is developed with examples by postulational methods, and the structure and periods of the associated cycles of marks are determined. Connections are established with the Galois field theory, diophantine analysis and the theory of ideals.

11. Mr. Morgan Ward: On the theory of ideal numbers. Preliminary report.

A theory of ideal numbers is developed and applied to cubic, quartic and certain cyclotomic fields. Its connection with Zolotareff's and Dedekind's theories and its relation to results in other branches of algebraic arithmetic is given.

12. Miss Marie J. Weiss: Primitive groups which contain substitutions of prime order $p$ and of degree $6 p$ or $7 p$.

In this paper the author proves that the degree of aprimitive permutation group (not alternating or symmetric) which contains a permutation of prime order $p(>7)$ and of degree $6 p(7 p)$ does not exceed $6 p+6(7 p+8)$.

B. A. Bernstein, Secretary of the Section

\title{
THE TWENTIETH REGULAR MEETING OF THE SOUTHWESTERN SECTION
}

The twentieth regular meeting of the Southwestern Section was held at Washington University on Saturday, November 26, 1927. The total attendance was thirty-five including the following twenty-two members:

Nola Anderson, Herman Betz, Florence Black, Brenke, Davisson, Dunkel, Gerst, Gouwens, Byron Ingold, Louis Ingold, Harry Levy, G. O. James, Jaeger, Osborn, Pennell, Rider, Edna Robinson, Roever, Eugene Stephens, Stouffer, J. S. Turner, Wyant.

Professor Roever occupied the chair being relieved during the sessions by Professor Brenke. The morning session was devoted to the reading of the papers listed below. During the afternoon session Professor E. B. Stouffer gave a special lecture by invitation of the program committee on Some canonical forms and associated canonical expansions in projective differential geometry. 
The titles and abstracts of the papers read are given below. The papers of Dr. Ayres and Professor Whyburn were read by title.

1. Professor W. C. Brenke: Polynomial solutions of a class of difference equations.

The author considers linear difference equations of the second order, of the form $y_{n}=\left(\alpha_{n}+\beta_{n} x\right) y_{n-1}-\gamma_{n} y_{n-2}$ where the coefficients $\alpha_{n}, \beta_{n}, \gamma_{n}$ are independent of the parameter $x$. Solutions of this equation which are polynomials in $x$, when such exist, are obtained in explicit form as special cases of the secular determinant. Many well known polynomials may thus be written as determinants.

2. Professor W. H. Roever: Some geometric relations concerning the theory of the Eötvös torsion balance. Preliminary communication.

In the weight field of force of the earth the lines of force have a first curvature which differs slightly from zero, and the level surfaces have, at each point, principal curvatures which differ slightly from each other. Small though these differences be, they determine those horizontal components of the weight which constitute the moments that operate the Eötvös torsion balance. By means of certain geometric theorems, which are proved in this paper, it is possible to determine directly, without any analytic treatment, expressions for the moments which operate the balance, and also to detect a relation which is usually overlooked in the theory of the balance.

3. Professor Florence Black: A reduced system of differential equations for the invariants of ternary forms.

A homogeneous function of the coefficients of a ternary form of order $n$ is invariant under the general linear transformation if and only if it is a solution of a certain complete system of nine linear partial differential equations of the first order. By the introduction of a set of new variables, F. Junker has reduced the number of differential equations in the complete system to seven. A comparison with the study made by E. B. Stouffer on invariants of linear homogeneous differential equations suggests a different choice of new variables. The author of the present paper has found that the introduction of these new variables serves to reduce the number of differential equations in the complete system to six. Moreover the resulting equations are far simpler in form than those obtained by Junker's reduction.

4. Professor Louis Ingold: Surfaces in five-dimensional space.

In this paper a vector function $f(u, v)$, depending on two scalar parameters, is considered. It is assumed that all third partial derivatives of 
the vector $f$ are linearly expressible in terms of the five first and second partial derivatives. It is shown how the coefficients in these partial differential equations for the vector $f$ may be expressed in terms of certain fundamental quantities which include the $E, F, G$ of ordinary differential geometry.

5. Professor G. O. James: Remarks on euclidean approximations to Einsteinian dynamics.

This paper contains a tentative examination of the assumptions made in approximating the Einsteinian motion of a particle in the solar field by a potential function in euclidean space.

\section{Mr. W. O. Pennell: Operational systems.}

This paper outlines several classes of operators which follow the fundamental laws of algebra, namely, the distributive, associative, and commutative laws and the index laws. The definition of the operators includes the interpretation of fractional exponents, as well as integral exponents. Multiplication and division is an operational process, and an example is given of algebraic division performed by an operational method. The name "gamma operator" is suggested for the operator whose definition equation is $p^{\alpha} x^{n}=\Gamma(n+1)^{1 / 2} x^{n-\alpha} / \Gamma(n-\alpha+1)^{1 / 2}$. This operator parallels, in most respects, the operation of differentiation and integration; it also defines fractional integration and differentiation. The important properties of this operator are briefly outlined and discussed.

7. Dr. W. L. Ayres: On continuous curves having certain properties.

In this paper it is proved: (1) if every arc of a plane continuous curve $M$ is a subset of a simple closed curve (an open curve) [a ray] of $M$ then $M$ is the entire plane or $M$ is a simple closed curve (every point of $M$ is a cutpoint) [every point of $M$ except possibly one is a cut-point]; (2) the boundary $B$ of a plane domain $D$ is uniformly regularly accessible from $D$ with respect to the bounded continua of $B$ if and only if (a) every component of $B$ is a point or a simple continuous curve, (b) if $J$ is any simple closed curve and $\epsilon>0$ is any number, then there are but a finite number of components of $B$ of diameter $>\epsilon$ that have points interior to $J ;(3)$ a continuum containing no cut-point is a simple closed curve if every two points of it may be separated by two points. A necessary and sufficient condition in order that a continuous curve contain an open curve is given.

\section{Professor G. T. Whyburn: Concerning Menger regular} curves.

The following are among the results proved in this paper. (1) If no maximal cyclic curve of a continuous curve $M$ contains an infinite collection of mutually exclusive simple closed curves, then (a) $M$ is a Menger regular curve which is a Baum im kleinen at all save a countable number of points, (b) the ramification points of $M$ are countable, and (c) every 
connected subset of $M$ is arcwise connected. (2) A cyclically connected continuous curve is a Baum im kleinen if and only if it has only a finite number of ramification points. (3) The ramification points of a continuous curve $M$ are countable if and only if the ramification points of each maximal cyclic curve of $M$ are countable. (4) If the set of all ramification points of a continuous curve $M$ is punctiform, then $M$ is a Menger regular curve. (5) If a bounded continuum $M$ is disconnected by the omission of any one of its countably infinite subsets, then $M$ is a Baum im kleinen. (6) A continuous curve $M$ is disconnected by the omission of any two of its points lying together on some simple closed curve in $M$ if and only if every maximal cyclic curve of $M$ is a simple closed curve.

\title{
9. Professor G. T. Whyburn; Accessibility theorems.
}

In this paper the author proves the following results. (1) If the limit point $P$ of a point set $R$ is not regularly accessible from $R$, then there exists a positive number $\epsilon$ and an infinite sequence of points of $R$ which has $P$ as a limit point and such that no two of these points can be joined in $R$ by any arc of diameter $<\epsilon$. (2) Every one dimensional (Menger-Urysohn sense) point $P$ of a continuum $M$ in $n(n \geqq 3)$ dimensions is regularly accessible from each complementary domain of $M$ whose boundary contains $P$. (3) In order that every point of a non-dense continuous curve $M$ in a plane $S$ should be regularly accessible from $S-M$ it is necessary and sufficient that $M$ should contain no infinite collection of mutually exclusive simple closed curves. (4) If $M$ is a plane non-dense continuous curve, $G$ denotes the collection of all the complementary domains of $M$, and $T$ denotes the limiting set of the collection $G$, then $T$ is identical with the set of all those points of $M$ at which $M$ is not a Baum im kleinen.

10. Professor J. S. Turner: On residues of iterated powers, modulo $m$.

In this paper it is proved that if $f$ belongs to $a$ (modulo $m$ ), and if $b$ is prime to $f$, then the residues of (I) $a, a^{b}, a^{b^{2}}, \cdots, a^{b^{f-2}}$, taken modulo $m$, are the same in the same order, as those of (II) $a, a^{2}, a^{3}, \cdots, a^{b-1}$, provided that $f$ is a prime, and $b$ is a primative root of $f$. In all other cases (I) contains fewer distinct residues than (II). Some consequences are pointed out.

\author{
E. B. Stouffer, \\ Secretary of the Section
}

